1992

\title{
Particulate release from surfaces exposed to a plasma
}

J. Goree

T.E. Sheridan

Follow this and additional works at: https://researchrepository.wvu.edu/faculty_publications

\section{Digital Commons Citation}

Goree, J. and Sheridan, T. E., "Particulate release from surfaces exposed to a plasma" (1992). Faculty Scholarship. 280.

https://researchrepository.wvu.edu/faculty_publications/280

This Article is brought to you for free and open access by The Research Repository @ WVU. It has been accepted for inclusion in Faculty Scholarship by an authorized administrator of The Research Repository@WVU. For more information, please contact ian.harmon@mail.wvu.edu. 


\title{
Particulate release from surfaces exposed to a plasma
}

\author{
J. Goree $e^{\text {a) }}$ \\ Department of Physics and Astronomy, The University of Iowa, lowa City, Iowa 52242 \\ T. E. Sheridan \\ Department of Physics, West Virginia University, Morgantown, West Virginia 26506
}

\begin{abstract}
(Received 18 May 1992; accepted 22 August 1992)
Contamination can occur during plasma processing when micrometer-size particulates fall from vacuum vessel walls onto a wafer. In situ light-scattering measurements show how particulates are shed from walls. Using a test surface coated with micron-size particles, we find that when a plasma is turned on, particulates are released rapidly, and when it is turned off, this release stops. This proves that plasma exposure causes particulate shedding. The rate of dust shedding increases with plasma density. The inventory of dust on the surface decays exponentially in time, with a time constant $\approx 10^{2} \mathrm{~s}$ in our experiment, for plasma densities of $\approx 10^{14} \mathrm{~m}^{\cdots 3}$. Particulates become negatively charged due to the flux of electrons and ions onto the surface and are then pulled off the surface by the electric field in the plasma sheath. An individual dust grain is shed when its charge $Q$ becomes sufficiently negative.
\end{abstract}

\section{INTRODUCTION}

In semiconductor manufacturing, considerable attention is given to controlling the particulate contamination that sometimes occurs during plasma processing. ${ }^{1-6}$ Particulates entering a plasma rapidly become negatively charged. They can be trapped in the plasma near electrode surfaces. ${ }^{5}$ Depending on the process and the tool, many of these particulates may eventually fall to a wafer and contaminate it. $^{2-5}$ The process of particulate contamination has several requirements. These include a source of particulates, a mechanism for transport to the trapping regions, the means of electrostatic charging and trapping, and finally the transport of contaminants either to the wafer or other surfaces.

There are several possible sources of particulates. They may grow by accretion in the gas phase after nucleating either on a surface or in the gas phase. ${ }^{6,7}$ Alternatively, contaminated surfaces such as vacuum vessel walls may release particulates full-grown into the plasma. This article examines the latter possibility.

Our experiment was designed to isolate the dust release process from competing processes that may be found, for example, in a reactive plasma etching environment. Rather than using an entire vacuum vessel wall, with an unknown history and unknown contaminants, we used a test surface that was deliberately coated with micrometer-sized dielectric particulates. And rather than detecting particulates on a wafer, after a process has been completed, we detected them in situ, as they were released, using time-resolved laser scattering. We find that particulate release is caused by plasma exposure, and its rate increases with plasma density. We also find that the inventory of dust on the contaminated surface decays exponentially in time. We attempt to quantify both the time scale and the charge required for a particulate to be released is attempted.

We have reported these results previously, Ref. 8 , in a paper written for spacecraft researchers concerned with particulate contamination in space plasmas. To make our results available to the plasma processing community, we provide here a synopsis of the experiment, in the context of contamination during semiconductor processing.

\section{APPARATUS}

\section{A. Test surface and particulates}

For the test surface, we used an aluminum sphere of diameter $4.45 \mathrm{~cm}$. This sphere serves as a proxy for a dirty vacuum vessel wall. It was deliberately covered with a freeflowing white powder, Alcoa tabular alumina $\left(\mathrm{Al}_{2} \mathrm{O}_{3}\right)$, having a dielectric constant ${ }^{9}$ that is $\geqslant 4.5$. Individual grains have a flaked surface, and sizes ranging from about $1 / 4$ to $10 \mu \mathrm{m}$, as shown in the electron micrograph in Fig. 1. The average mass of a grain, estimated from electron micrographs, is about $0.3 \mathrm{ng}$.

The test surface was prepared in air before insertion in the vacuum vessel. The surface was first cleaned with isopropyl alcohol, and then alumina particulates were applied electrostatically. The sphere was charged using a Van de Graaff generator and was then moved over a powdercovered glass plate so that electrostatic attraction transferrer alumina dust from the plate to the sphere. After applying the dust, we grounded the sphere to remove any net charge. The powder remained attached to the surface by adhesion. This procedure yielded a fairly uniform coating. By weighing the dust scraped off the sphere, we found that $80 \pm 10 \mathrm{mg}$ of alumina dust had been applied, containing roughly $2 \times 10^{8}$ individual grains. We applied such a large quantity to assure a strong laser scattering signal.

The sphere was mounted on a motor-drive shaft that rotated at $9.9 \mathrm{rpm}$. We found that rotation was necessary so that the plasma did not remove dust preferentially from a single area of the sphere. The sphere was grounded through a large 4-M $\Omega$ impedance so that it drew no significant current from the plasma. That is, the surface was at the floating potential $V_{\text {float }}$, which we recorded during the experiment. 


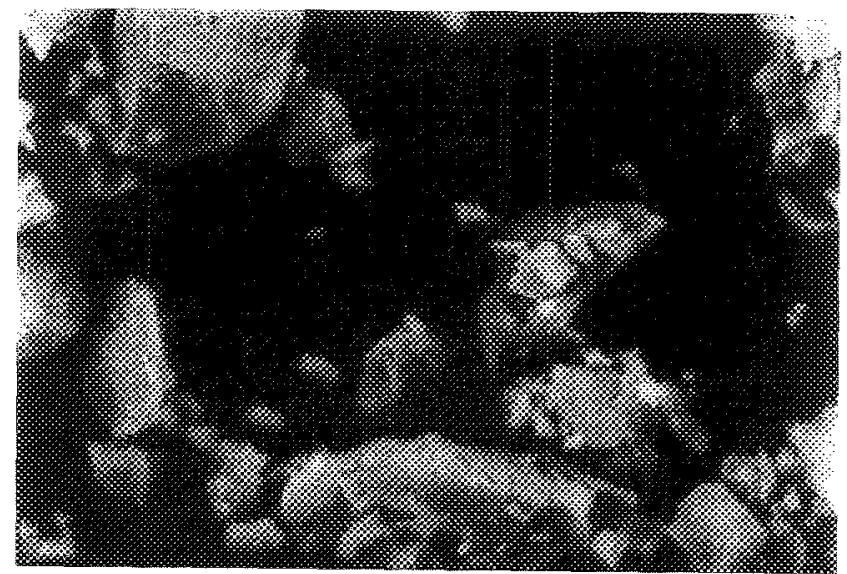

FiG. 1. Electron micrograph of alumina $\left(\mathrm{Al}_{2} \mathrm{O}_{3}\right)$ particulates. The calibration bar is $10 \mu \mathrm{m}$ long. From pictures taken with a lower magnification, we determined that the average grain size is approkimately $8 \mu \mathrm{m}$. Individual grains are tabular and have a fractured surface.

In contrast to a dirty wall in a processing tool, which will probably be grounded, our test surface was floating. Measured with respect to the plasma potential, a grounded wall is at a potential $-V_{\text {plasmar }}$, while our test surface floated at $\phi=V_{\text {fleat }}-V_{\text {plasma. }}$. This is not ordinarily a significant distinction. In our experiment, $\phi \approx-20 \mathrm{~V}$, so only when $V_{\text {plasma }}$ is radically different from $20 \mathrm{~V}$ would our results be inapplicable to grounded walls.

\section{B. Vacuum system and plasma}

The experiment was performed in the $26-\ell$ cylindrical vacuum vessel shown in Fig. 2. The vessel is aluminum, black anodized to reduce scattered light. It is evacuated by a turbomolecular pump to a base pressure of $8 \times 10^{-5} \mathrm{~Pa}$. For plasma operation, we admitted nitrogen gas into the vessel through a piezoelectric valve, regulated to maintain a constant $0.056 \mathrm{~Pa}$ pressure.

A de plasma was created in a source chamber with hot filaments and muitidipole magnetic confinement. ${ }^{10}$ The source was separated from the main vessel by a grounded grid. To provide a ground reference for the plasma, a stainless-steel anode plate was placed at the bottom of the vessel. The plasma filled the entire vacuum vessel. Since the filaments are heated and biased independentiy, there are two ways to control the discharge, the filament temperature and the discharge voltage $V_{\text {dis. }}$.

The discharge current $I_{\text {dis }}$ drawn between the filaments and the grounded vacuum vessel serves as a rough indication of the plasma density $n_{e}$. These two quantities are proportional, $I_{\mathrm{dis}} \propto n_{n}$, provided that the electron temperature is approximately constant, which it often is. To characterize $n_{e}$ and the other plasma parameters, we used a small cylindrical Langmuir probe. For two of the discharges used in the shedding experiment, the parameters were as follows. For $V_{\text {dis }}=-40 \mathrm{~V}$ and $I_{\mathrm{dis}}=1.0 \mathrm{~A}$, the electron temperature and density were $T_{e} \approx 7.3 \mathrm{eV}$ and $n_{e} \approx 10^{13} \mathrm{~m}^{-3}$, respectively. At this low discharge voltage, the electron distribution is somewhat non-Maxwellian. For

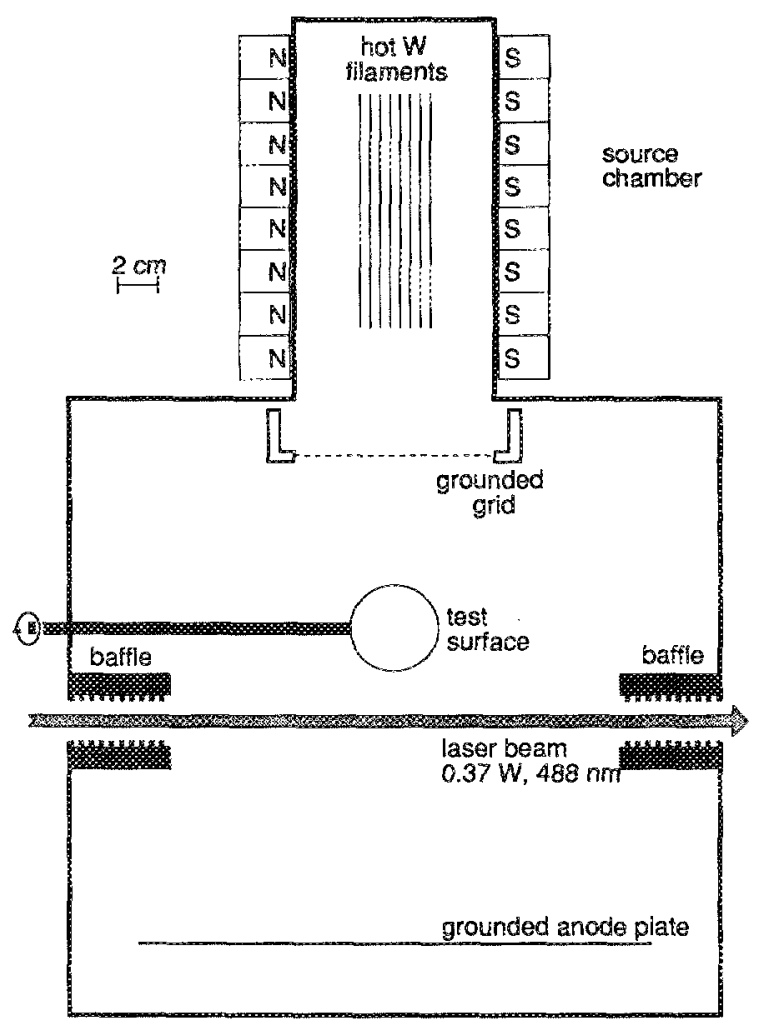

FIG. 2. Side view of the apparatus. A nitrogen palsma, produced by electrons from a hot filament source, fills the entire vessel. Dust falling from the spherical test surface is detected by laser light scattering.

$V_{\mathrm{dis}}=-60 \mathrm{~V}$ and $I_{\text {dis }}=1.0 \mathrm{~A}$, we found $V_{\text {plasma }}=5 \mathrm{~V}$, $T_{e}=4.1 \mathrm{eV}$ and $n_{e}=1.1 \times 10^{14} \mathrm{~m}^{-3}$.

Plasma from this type of source usually have two electron components: a primary component emitted directly by the filaments, and a denser, cold component of secondary electrons. In the probe characteristics, we found the fast component to be noticeable at lower discharge voltages, $V_{\text {dis }} \approx-40 \mathrm{~V}$. Finally, we note that raising the discharge voltage increased the plasma density significantly.

\section{Light scattering}

To make in situ, time-resolved measurements of the dust shedding, we used laser-light scattering. The optical layout is shown in Figs. 2 and 3. A 488-nm Ar laser, operating steady-state at $0.37 \mathrm{~W}$, was aimed beneath the dustcovered sphere. Mie scattering of laser light from particulates falling of the sphere was detected at $45^{\circ}$ from the forward direction. The detection optics consisted of a lens $(150 \mathrm{~mm}$ focal length) and an aperture $(5.0 \mathrm{-mm}$ diam), aligned to locate the detection volume directly beneath the test sphere. To reduce stray laser light, we installed baffies at the input and output windows. Stray light from other sources, especially the hot flaments, was reduced using a 488-nm bandpass filter, with a 3-nm bandwidth and $55 \%$ maximum transmission. Not all of the white light could be blocked, and this contributed to a baseline in our signal that increased with filament temperature. The filtered light was detected by a photomultiplier tube (PMT), which produced a current proportional to the amount of light scat- 


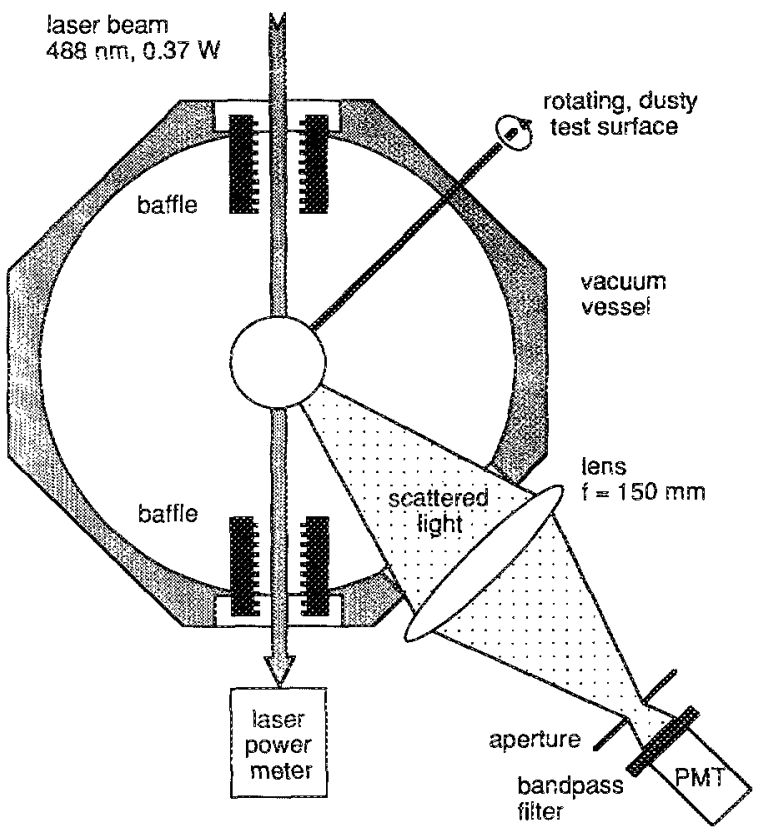

FIG. 3. Top view of the light scattering diagnostic. Laser light $(\lambda=488$ $\mathrm{nm}$ ) is collected at $45^{\circ}$ from the forward direction by a $10-\mathrm{cm}$-diam lens with a focal length of $150 \mathrm{~mm}$. A $5.0 \mathrm{-mm}$ diam circular aperture at the focal point defines the volume from which scattered light is collected. A 488-nm optical bandpass filter rejects white light. Scattered light is detected by a photomultiplier tube (PNT).

tered. Hereafter we refer to this current as the "scattered light signal." It was sampled approximately twice per second. (We also recorded $I_{\text {dis }}, V_{\text {dis, }}$, and $V_{\text {float }}$; these measurements are reported in more detail in Ref. 8.)

\section{EXPERIMENT}

We measured dust shedding under a variety of plasma conditions. The time history of the scattered light signal is shown in Fig. 4. In examining Fig. 4, one should look for spikes in the scattered light signal, indicating light scattered from falling dust grains.

At the start of the experiment, the source filaments were turned on without making a plasma. The signal in Fig. 4 increased to a steady baseline level due to white light from the filaments. This baseline should not be confused with dust shedding. The lack of shedding at this time shows that heat from the filaments does not precipitate dust release.

A plasma was formed at $116 \mathrm{~s}$ by augmenting the discharge voltage to $-40.3 \mathrm{~V}$. A small scattered light signal is evident above the baseline, indicating weak shedding. Here the plasma parameters are $I_{\text {dis }}=0.71 \mathrm{~A}, T_{e}=7 \mathrm{eV}$, $n_{c}=7 \times 10^{12} \mathrm{~m}^{-3}$, and $V_{\text {lioat }}=-17 \mathrm{~V}$.

Rotation of the sphere began at $140 \mathrm{~s}$. This caused the scattered light signal to increase, as fresh areas on the surface were brought under the plasma source, yielding a larger dust release. Additionally, the scattered light was modulated at the roration frequency, due to slightly different rates of shedding from different areas on the sphere.

The next step provides direct evidence that the plasma is responsible for the observed dust shedding. At $190 \mathrm{~s}$ we turned the plasma off by turning the discharge voltage to

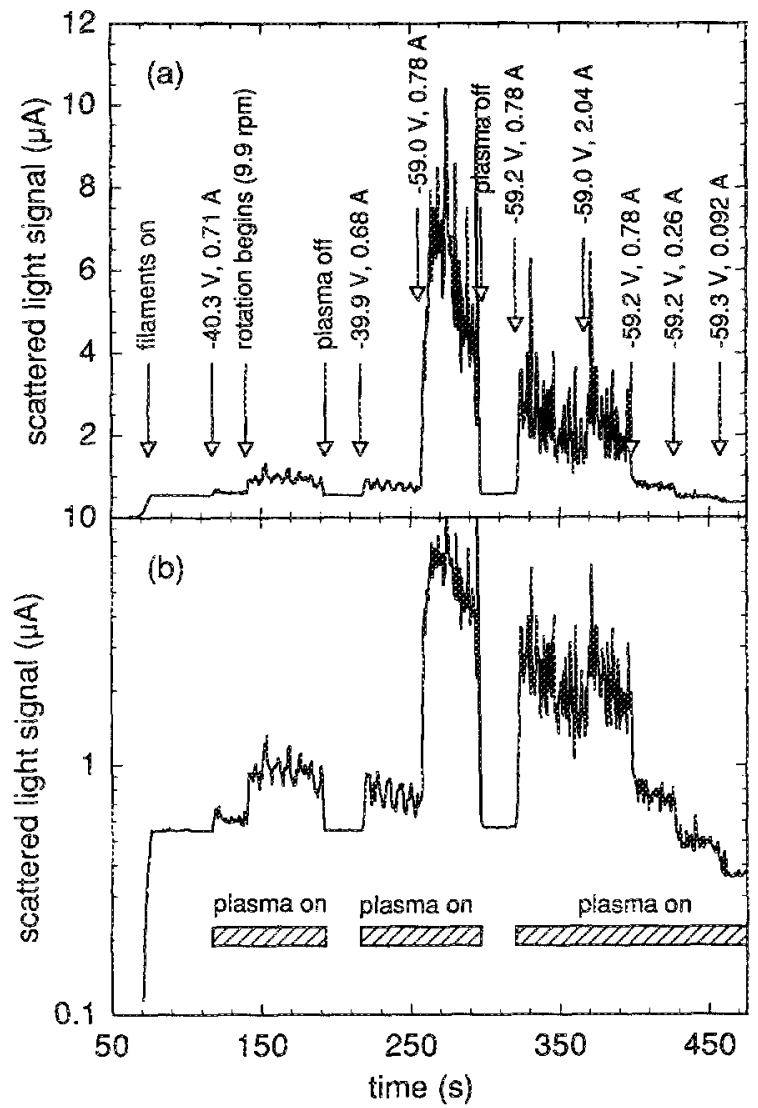

FIG. 4. Time history of the scattered light signal. The data is plotted (a) on linear axes, and (b) on semilogarithmic axes. During the plasma-on intervals, we operated at several different discharge voltages and currents, consequently the plasma-on periods are not identical. Changes in the plasma conditions are marked in (a) and summarized in Table I. The baseline is due to light from the hot filaments. Dust shedding is indicated by spikes above the baseline. Dust shedding occurs only when the plasma is on.

zero. The scattered light signal returned to its baseline value, indicating that shedding had stopped. When the plasma was restarted at $230 \mathrm{~s}$ with nearly the same parameters, dust shedding resumed at about the same level as before.

This is the chief result of the article. When the plasma is on, there is shedding, and when it is off, the shedding stops. For the remainder of the experiment, we explored the dependence of shedding on differing plasma conditions, as summarized in Table I. These results are reviewed next.

We found that the rate of shedding increases with plasma density. This is seen in Fig. 4 at $256 \mathrm{~s}$, when the discharge voltage was augmented to $-59.0 \mathrm{~V}$. This caused the plasma density to increase by approximately an order of magnitude to $8.6 \times 10^{13} \mathrm{~m}^{-3}$. The discharge current increased only slightly to $0.78 \mathrm{~A}$, and the electron temperature decreases to about $4 \mathrm{eV}$. Under these conditions, the scattered light signal grew large, indicating a high rate of shedding. The scattered light signal then diminished slowly with time as dust was depleted from the test surface.

To isolate the dependence of shedding rate on plasma density, we varied $n_{e}$ while holding $V_{\mathrm{dis}}$ constant. Since $n_{e} \propto I_{\text {dis }}$ for a constant $V_{\text {dis }}$, we varied the density by ad- 
TABLE I. Time history of the experiment. Electron density $n_{e}$ and temperature $T_{e}$ are listed for the discharges where they were measured. Values are given here for $I_{\text {dis }}$ and $V_{\text {fioat }}$ are time averages; their time evolution is presented in Ref. 8 .

\begin{tabular}{|c|c|c|c|c|c|c|}
\hline $\begin{array}{l}\text { Time } \\
\text { (s) }\end{array}$ & Procedure & $\begin{array}{l}y_{\text {dis }} \\
\text { (v) }\end{array}$ & $\begin{array}{l}I_{\text {dis }} \\
(\mathrm{A})\end{array}$ & $\begin{array}{l}V_{\text {foat }} \\
(\mathrm{V})\end{array}$ & $\left(10^{63} \mathrm{~mm}^{n_{e}}-3\right)$ & $\begin{array}{c}T_{c} \\
(\mathrm{eV})\end{array}$ \\
\hline 66 & Filaments on & 0 & 0 & & 0 & 0 \\
\hline 116 & Plasma on & -40.3 & 0.71 & -17 & 0.7 & 7 \\
\hline 140 & Rotation begins & -40.3 & 0.71 & -17 & 0.7 & 7 \\
\hline 190 & Plasma of & & & & & \\
\hline 230 & Plasma on & -39.9 & 0.68 & -18 & & \\
\hline 256 & $V_{\text {dis }}$ increased & -59.0 & 0.78 & -15 & 8.6 & 4 \\
\hline 295 & Plasma of & & & & & \\
\hline 319 & Plasma on & -59.2 & 0.78 & -16 & & \\
\hline 368 & $I_{\text {dis }}$ increased & -59.2 & 2.0 & -22 & & \\
\hline 398 & $I_{\text {dis }}$ refuced & -59.2 & 0.78 & -20 & & \\
\hline 428 & $I_{\text {crs }}$ reduced & -59.2 & 0.26 & -18 & & \\
\hline 466 & $I_{\text {dis }}$ reduced & -59.2 & 0.092 & $\ldots 16$ & & \\
\hline
\end{tabular}

justing the discharge current via the filament temperature. (Changing the filament temperature also has the unfortunate side-effect of shifting the baseline of the scattered light signal.) This sequence begins at $t=368 \mathrm{~s}$. Figure 4 shows that each time $I_{\text {dis }}$ was reduced, the shedding rate diminished. This confirms our conclusion that dust shedding increases with plasma density.

After the plasma experiment was completed, the sphere was removed from the vacuum vessel. The amount of dust remaining on it was $50 \pm 10 \mathrm{mg}$. This means that half of the dust had been scoured from the surface after a few minutes of plasma exposure. From this we can estimate, within an order of magnitude, the dust shedding rate. About $10^{8}$ grains were shed while the plasma was on, indicating a rate of about $10^{6}$ grains $\mathrm{s}^{-1}$, averaged over the entire experiment. This gives a shedding rate per unit area of approximately $2 \times 10^{8}$ grains s $^{-1} \mathrm{~m}^{-2}$.

In addition to our conclusion that shedding increases with plasma density, we can also say that it is augmented by the presence of fast electrons in the plasma. The latter finding was made by visual cbservations of shedding from the test surface. We saw shedding from the entire surface of the sphere, but it was strongest on the top, which was exposed directly to the flaments. This is likely due to the downward fux of primary electrons from those filaments.

\section{DISCUSSION}

\section{A. Forces acting on a particulate}

The experiment described above proves that plasma exposure causes dust shedding from a soid surface. When the plasma is on, there is shedding, and when it is off, the shedding stops. Having established this, we now attempt to identify the basic physics relevant to the shedding process.

We first identify the forces acting on a particulate while it is attacined to the surface. They are adhesive, electrostatic, and gravitational, as sketched in Fig. 5. The centrifigual force due to rotation was negligible, as if was four orders of magnitude weaker than gravity. Chemical adhesive forces bind the particulates in varying degrees to the

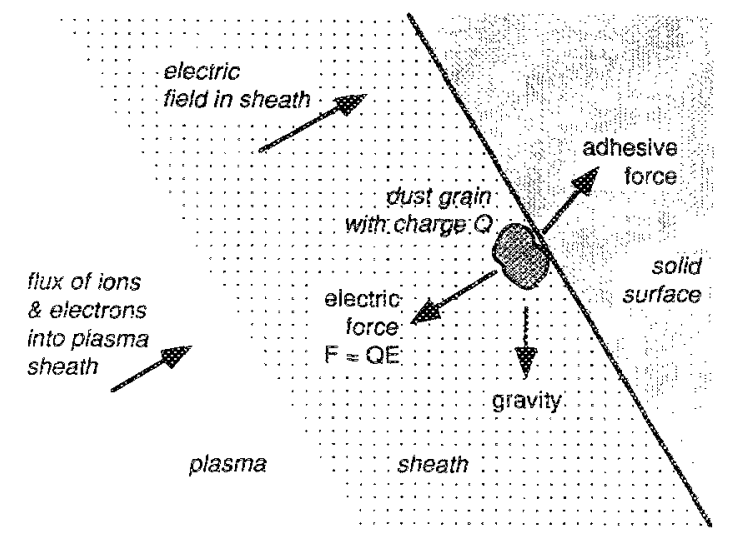

Fics. 5. Sketch of the forces acting on a dust grain attached to a surface. It is not necessary that the surface be inverted, as drawn here, for the grain to be released into the plasma. The electric field in the plasma sheath tends to pull the negatively charged particulite from the surface.

surface. We cannot say much about this force, except that it was stronger than gravity alone, since the sphere was turned upside down many times while preparing it, and the dust remained attached. The adhesive force is presumably proportional to surface area $a^{2}$, where $a$ is the characteristic size of a given particulate. The mass, and thus the gravitational force, scales with the volume of the dust grain, $a^{3}$. The electric force scales with $a$, as shown below. These three scaling laws suggest that gravity tends to pull of the largest grains from an inverted surface, while the electric force removes the smallest grains.

The electric force $Q E$ arises when the particulate and the surface are exposed to a plasma. Here $E$ is the electric field at the surface due to the plasma sheath, and $Q$ is the net charge accumulated on a particulate by collecting plasma ions and electrons. The direction of $E$ is toward the surface, since the surface is at a negative potentiai $\phi$ with respect to the plasma. This direction is important because it means that the electric force on a negative charged particulate will be away from the surface, thereby promoting shedding. The magntiude of $E$ can be roughily estimated as $\left(k T_{e} / e\right) / \lambda_{D}$, since the potential drop is on the order of $-k T_{e} / e_{2}$ over one Debye length $\lambda_{D}$. More exactly, at a planar surface $E$ is given by ${ }^{11}$

$$
E=\frac{k T_{e} / e}{\lambda_{D}} 2^{1 / 2}\left[\sqrt{1-\frac{2 e \phi}{k T_{e}}}+\exp \left(\frac{e \phi}{k T_{e}}\right)-2\right]^{1 / 2}
$$

valid for a Maxwelian plasma, where $\phi$ is the surface potential measured with respect to $V_{\text {plasma. }}$ (For this experiment the sheath widh is small compared to the radius of the test sphere, so that the sheath is locally planar.) In our experiment, the electric field computed from Eq. (1) is $E=41 \mathrm{~V} / \mathrm{cm}$ for $T_{e}=4 \mathrm{eV}, \phi=-20 \mathrm{~V}, n_{g}=8.6 \times 10^{13}$ $\mathrm{m}^{-3}$, and $\lambda_{D}=1.6 \mathrm{~mm}$, which are for a discharge operated at $V_{\text {dis }}=-60 \mathrm{~V}$ and $I_{\text {dis }}=0.78 \mathrm{~A}$.

The particulate gains a charge $Q$ while attached to the surface. This charge arises from the ion and electron fuxes entering the sheath and striking the surface. The dust particle collects a fraction of the incigent ions and electrons. This charges the particle to a value $Q=C V_{\text {grain }}$, where $C$ is 
the particle's capacitance and $V_{\text {grain }}$ is its potential with respect to the local plasma potential. For a spherical particulate of radius $a, C=4 \pi \epsilon_{0} a$. Both the charge and the potential will generally be negative, for the same reason that the floating potential of the sphere is negative: fast electrons must be repelled so that their current balances that of the slower ions. Consequently, the electric force tends to pull particulates from contaminated surfaces.

\section{B. Experimental estimate of $Q$}

The charge $Q$ of a particle while it is on the surface may be roughly estimated by comparing the electrical force to the gravitational force. We know from visual observations that dust was often shed from the top of the sphere. This indicates that the electric force $Q E$ was greater than the gravitational force, i.e., $|Q| E>m g$, where $m$ is the mass of a particulate. This upward levitation allows us to estimate a iower limit on $|Q|$. It is only a lower limit and not an equality, because the adhesive force is unknown. For the case of an $8-\mu \mathrm{m}$ diam spherical particle, we find $|Q|$ $>15000 e$, where $e$ is the elementary charge. Smaller particles would have a smaller charge.

As a check on this result, we can use the capacitance $C=4 \pi \epsilon_{0} a$ to compute the potential of an $8-\mu \mathrm{m}$ particle. This yields $V_{\text {grain }} \approx-5 \mathrm{~V}$, which is in order of magnitude agreement with the floating potential $\phi=-20 \mathrm{~V}$ of the aluminum sphere.

It is revealing that the charge $Q$ can attain such a large value. One might have expected that the charge on a single dust particle would be merely a fraction of the total charge on the whole test surface, in the ratio of its area to the test surface area. But a simple calculation shows that if this were so, $Q$ would be usually either zero or one electron charge, too small for shedding. Instead, the dielectric particle acquires a net charge of several thousand $e$, indicating that it acts like a small capacitor of its own, without efficiently conducting its charge to the surface.

\section{Time scale for shedding}

We find that the number of dust grains remaining on the surface decays exponentially in time, under constant plasma conditions. This is demonstrated by the linear decrease in the scattered light signal seen in Fig. 4(b), with semilogarithmic axes, during intervals when the plasma parameters were held steady. Grains are shed from the surface at a rate that is proportional to the number remaining. The rate of shedding decreases in time, as the amount of dust on the surface is depleted.

The time constant $\tau_{1 / \text { e }}$ for the dust depletion is about $10^{2} \mathrm{~s}$, averaged over all the operating conditions in our experiment. At higher plasma densities, $\tau_{1 / e}$ is shorter. It may also vary with the dust composition and surface his- tory. Knowing this time scale may be useful to the reader planning a process, as it may help to know how rapidly particulate contamination will take place after the plasma is first turned on.

The exponential decay indicates that the shedding process occurs with individual grains jumping off at random intervais. The probability per unit time of one grain jumping off remains roughly constant provided that the plasma conditions do not change. This result in interesting, because it rules out the possibility that the particulates might all be shed in a single burst when the plasma is first turned on. The random nature of the dust shedding indicates that the release mechanism of the grains from the surface depends on a quantity that varies statistically with time.

\section{SUMMARY}

We find that particulates are steadily and rapidly removed from a surface exposed to a plasma. This occurs due to the charge $Q$ of the particulates and a sheath electric field $E$ at the surface. Individual grains are shed at random intervals, with a probability per unit time that increases with plasma density. The inventory of dust remaining on the surface decays exponentially in time, with a time constant on the order of $10^{2} \mathrm{~s}$. These results should be useful in understanding the evolution of particulate contamination from walls in plasma processing tools.

\section{ACKNOWLEDGMENTS}

This work was supported by Lockheed Missiles and Space Company, under Contract No. 605352-L from The Applied Physics Laboratory of the Johns Hopkins University.

2) Temporary address: Max-Planck-Institut für extraterrestrische Physik, 8046 Garching, Germany.

${ }^{1}$ R. M. Roth, K. G. Spears, G. D. Stein, and G. Wong, Appl. Phys. Lett. 46, 253 (1985).

${ }^{2}$ G. S. Selwyn, J. Singh and R. S. Bennett, J. Vac. Sci. Technol. A 7, 2758 (1989).

${ }^{3}$ G. S. Selwyn, J. E. Heidenreich, and K. L. Haller, Appl. Phys. Lett. 57, $1876(1990)$.

${ }^{4}$ G. S. Selwyn, J. S. McKillop, K. L. Haller, and J. J. Wu, J. Vac. Sci. Technol. A 8, 1726 (1990).

${ }^{5}$ M. S. Barnes, J. H. Keller, J. C. Forster, J. A. O'Neill, and D. K. Coultas, Phys. Rev. Lett. 68, 313 (1992).

${ }^{6}$ M. M. Smadi, G. Y. Kong, R. N. Carlile, and S. E. Beck, J. Vac. Sci. Technol. B 10, 30 (1992).

${ }^{7}$ H. M. Anderson, R. Jairath, and J. L. Mock, J. Appl. Phys. 67, 3999 (1990).

${ }^{8}$ T. E. Sheridan, J. Goree, Y. T. Chiu, R. L. Rairden, and J. A. Kiessling, J. Geophys. Res. Space Phys. 97, 2935 (1992).

${ }^{9} \mathrm{CRC}$ Handbook of Chemistry and Physics, edited by R. C. Weast (Chemical Rubber, Boca Raton, FL, 1978), p. E-60.

${ }^{10} \mathrm{R}$. Limpaecher and K. R. MacKenzie, Rev. Sci. Instrum. 44, 726 (1973).

${ }^{11}$ F. F. Chen, Introduction to Plasma Physics (Plenum, New York, 1974), p. 246. 